Vol. LXIV No 12014

\title{
KNOWLEDGE REPOSITORY FOR FMEA RELATED KNOWLEDGE
}

\author{
GABRIELA SIMONA CÂNDEA \\ Faculty of Engineering /Department Engineering and Management, "Lucian Blaga" University, Sibiu, \\ Romania,gabriela.candea@ropardo.ro \\ CLAUDIU VASILE KIFOR \\ Faculty of Engineering /Department Engineering and Management, "Lucian Blaga" University, Sibiu, \\ Romania, claudiu.kifor@ulbsibiu.ro \\ CIPRIAN CÂNDEA \\ ROPARDO S.R.L., Sibiu, Romania, ciprian.candea@ropardo.ro
}

\begin{abstract}
This paper presents innovative usage of knowledge system into Failure Mode and Effects Analysis (FMEA) process using the ontology to represent the knowledge. Knowledge system is built to serve multi-projects work that nowadays are in place in any manufacturing or services provider, and knowledge must be retained and reused at the company level and not only at project level. The system is following the FMEA methodology and the validation of the concept is compliant with the automotive industry standards published by Automotive Industry Action Group, and not only. Collaboration is assured trough web-based GUI that supports multiple users access at any time.
\end{abstract}

Keywords: FMEA, knowledge capitalization, ontology

\section{Introduction}

The Failure Mode and Effect Analysis (FMEA) is one of the procedures that allow preventing the problems before they occur. Used in both the design and manufacturing processes, they substantially reduce costs by identifying product and process improvements early in the develop process when changes are relatively easy and inexpensive to make. The result is a more robust process/product because the need for after-the-fact corrective action and late change crises are reduced and eliminated (McDermott, Mikulak and Beauregard, 2008).

This paper proposes an ontology-based FMEA system which aims to extract and convert the FMEA related knowledge.

The paper start with presentation of the FMEA components followed by FMEA challenges collected from engineers involved in manufacturing processes. The approach used in order to achieve the objective is presented in the chapter 4 and the ontology and knowledge repository for FMEA related knowledge is presented in chapter 5 .

Although the purpose, terminology and other details can vary according to type (e.g. Process FMEA - PFMEA, Design FMEA - DFMEA, System FMEA, Product FMEA, FMECA, etc.), the basic methodology is similar for all one common factor has remained through the years - to resolve potential problems before they occur. For years, FMEA/FMECA has been an integral part of engineering designs. For the most part, it has been a necessary tool for industries such as the aerospace and automotive industries.

There are a number of published guidelines and standards for the requirements and recommended reporting format of failure mode and effects analyses. Some of the main published standards for this type of analysis include SAE J1739, AIAG FMEA-4 and MIL-STD-1629A. In addition, many industries and 
companies have developed their own procedures to meet the specific requirements of their products/processes.

FMEA/FMECA is a group activity (normally with 6-10 members) and may be done in more than one sitting, if necessary, following few steps (see Figure 1):

- detect a failure mode,

- grade the severity of this potential failure,

- grade the probability of this potential failure to happened,

- grade the easiness $f$ detection of this potential failure

- calculate the Risk priority number, and

- take actions and do checks.

The process owner (or project manager) is normally the leader of the FMEA exercise, however to get best results she is expected to involve multi-disciplinary representatives from all affected activities. Team members should include subject matter experts and advisors as appropriate. Each Process Owner is also responsible for keeping the FMEA updated.

Figure 1 - Main steps of FMEA

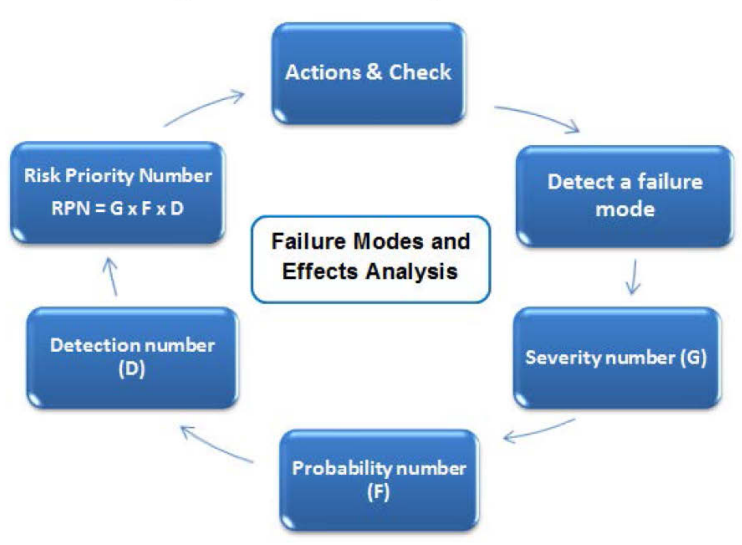

A good FMEA can help analysts identify known and potential failure modes and their causes and effects, assess the risk associated with those failure modes, prioritize the identified failure modes and identify and carry out corrective actions. The priority of a failure mode is determined through the risk priority number $(\mathrm{RPN})$, which is defined as the product of the occurrence $(\mathrm{O})$, severity $(\mathrm{S})$ and detection (D).

FMEA has been proven to be one of the most important early preventative initiatives during the design stage of a system, product, process or service. However, the big number of experienced people (from different departments) requested, the hardly reusable analyses because of the natural language, the unavailability (de-located team, overlap of membership between the teams) of team members represents the red line for the presented research.

\section{FMEA components}

'Failure modes' means the ways, or modes, in which something might fail. Failures are any errors or defects, especially ones that affect the customer, and can be potential or actual.

FMEAs are developed in very distinct phases where actions can be determined (Tague 2004). It is also required to do pre-work ahead of the FMEA to assure that the robustness and past history are included in your analysis.

On short, there are three components that help define the priority of failures:

- Occurrence (O)

- Severity of effect (S)

- Detection (D) 
Occurrence is the assessment of the probability that the specific cause of the Failure mode will occur. Severity is an assessment of the seriousness of the Effect and refers directly to the potential failure mode being studied.

Detection is an assessment of the probability that the proposed Process Controls will detect a potential cause of Failure or a Process weakness.

All FMEA processes follow these steps and the result can be seen in the Figure 2:

- Process Review.

- Brainstorm Potential Failure Modes.

- List Potential Effects of Each Failure Mode.

- Assigning Severity, Occurrence and Detection Ratings.

- Assign a Severity Rating for Each Effect.

- Assign an Occurrence Rating for Each Failure Mode.

- Assign a Detection Rating for Each Failure Mode and/or Effect.

- Calculate the Risk Priority Number for Each Failure Mode.

- Prioritize the Failure Modes for Action

- Take Action to Eliminate or Reduce the High-Risk Failure Modes

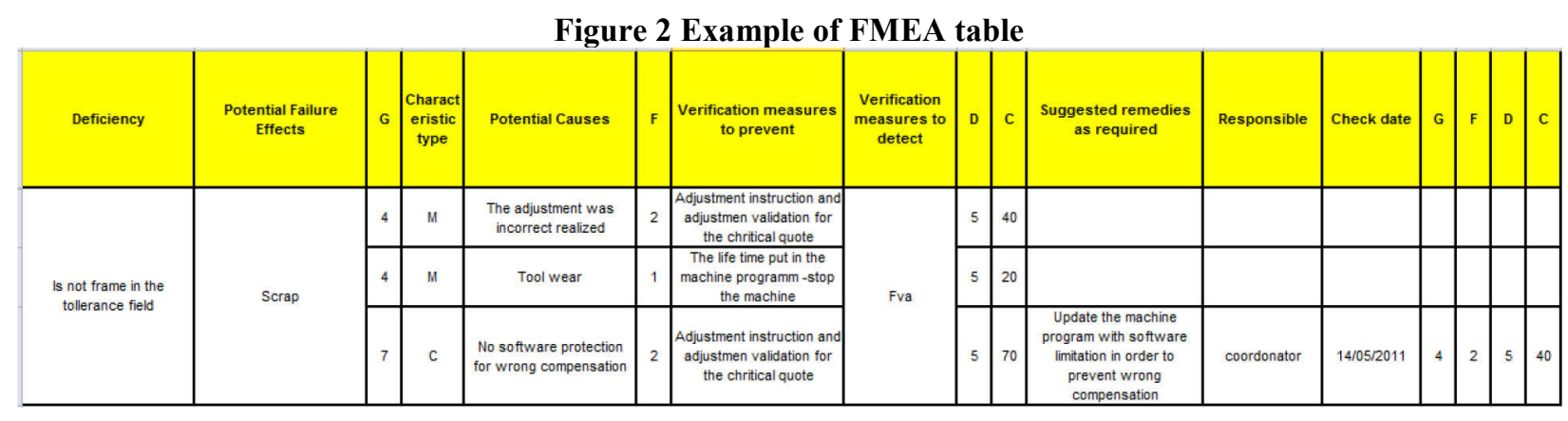

\section{FMEA challenges}

Today, FMEA is in widespread use by a multitude of industries, many of which have begun imposing FMEA standards. The purpose of the FMEA is to take actions to eliminate or reduce failures, starting with the highest-priority ones (Tague, 2004). But the effort to develop an FMEA is mainly considered as high or very high due to the number of involved persons (Stock, et all, 2003). In addition, the advantages that result out of failure prevention cannot be perceived immediately. To shorten the process of FMEA development and earning results, the knowledge included in already developed FMEA has to be reused and the first step is to capitalize the knowledge. The capitalization will not just shorten the FMEA process but will prevent that valuable job-related information will leave the company together with the employee.

The FMEA knowledge reuse endures from a major short-coming mentioned by Wirth et al. (1996): the FMEA-related information is acquired in natural language and is not much reusable because the systematized components, functions and failure modes are not made explicit. The meaning depends on the interpretation of the team/ a team member who performs the FMEA and can fluctuate when another team reuses this FMEA, or even if the same team tries to reuse it on a later occasion.

Although one person is in charge for coordinating the FMEA process, all FMEAs are team based. The scope for a FMEA team is to gather a range of perspectives and experiences in the project. Because each FMEA is unique in dealing with different aspects of the product or process (production, engineering, logistic, marketing, support), FMEA teams are formed and dispersed when is needed. Based on this variety of requested people another short-coming is sets by the unavailability (de-located team, overlap of membership between the teams) of team members to attend at FMEA meeting. 


\section{Our approach}

In this chapter the software FMEA system components are presented. The FMEA system is composed by two major subsystems - FMEA software and knowledge repository.

FMEA software is respecting all FMEA requirements and processes of work and is a web-based software that allows collaborative work for team members.

Second major system is Knowledge Repository System that provides knowledge capitalization for FMEA. In current implementation of Knowledge Repository System it's used for capitalization of knowledge a case base reasoning (CBR) approach.

Based on the analyses made about the shortcomings of FMEA methods the FMEA system offers the following features and benefits:

- Web application, can be access it via Internet based on assigned rights

- Has implemented Experience Database (Knowledge Repository System)

- Has Case base reasoning (CBR) approach.

- Use of nomenclatures

- Multi-user application

The experience database is proposed to provide an easy to use component by the knowledge engineer and by other software modules. The Experience Database component that is the main architecture is using Case-based Reasoning as computational engine. Actual design of Experience Database allows defining and storing different types of structures for knowledge representation. These structures can be defined using ontology editor that allow you to keep an organized and easy way to access and view of the database. In this case I used Protégé as ontology editor and defined ontology is stored using Protégé internal storage.

In this implementation of Experience Database, a Case Base Reasoning engine is the core computational engine that solves problems by adapting solutions to older ones (Cândea, 2012a).

A CBR system involves reasoning from prior examples, memorizing previous problems and associated solutions and solving new problems by referencing to that knowledge.

The problem-solving life cycle in our CBR system consists essentially of the following four parts:

- Retrieving similar previously experienced cases (e.g., problem-solution-outcome triples) whose problem is judged to be similar

- Reusing the cases by copying or integrating the solutions from the cases retrieved

- Revising or adapting the solution(s) retrieved in an attempt to solve the new problem

- Retaining the new solution once it has been confirmed or validated.

\section{Ontology Repository for FMEA related knowledge}

In Computer Science, "An ontology is a formal explicit description of concepts in a domain of discourse (classes - sometimes called concepts), properties of each concept describing various features and attributes of the concept (slots - sometimes called roles or properties), and restrictions on slots (facets - sometimes called role restrictions). One ontology together with a set of individual instances of classes constitutes a knowledge base. Ontology represents a common vocabulary, for users who need to share and exchange information in a domain. It embodies machine-interpretable definitions of basic concepts in the domain and relations among them. (Cândea, 2012)

As defined by Wikipedia: "Ontology is a formal representation of a set of concepts within a domain and the relationships between those concepts. It is used to reason about the properties of that domain, and may be used to define the domain".

As noted in Dittmann et all, 2004 and Laaroussi, et all, 2007, ontology can support the development and performance of an FMEA in two ways:

- First, it offers a common understanding of the concepts of the domain of our focus and the FMEA procedure ourselves as well. 
- Second, the knowledge held in the ontology based model can be computationally processed.

Both these basic facts support the idea to use an ontological approach for improvement of the FMEA procedure.

The main contribution of ontology based approach is a clear and consistent graphical description and visual presentation of all FMEA concepts and dependencies.

In order to create FMEA domain ontology repository in a structured way, in this paper it's used UML (Uniform Modelling Language), a class diagram, to express the structural model of FMEA domain knowledge. Uniform Modelling Language (UML) is a standardized (ISO/EC 19501:2005) visual modelling language used to in software engineering and includes a set of graphic notation to create visual models (UML, online).

Figure 3 Hierarchical relation of FMEA knowledge

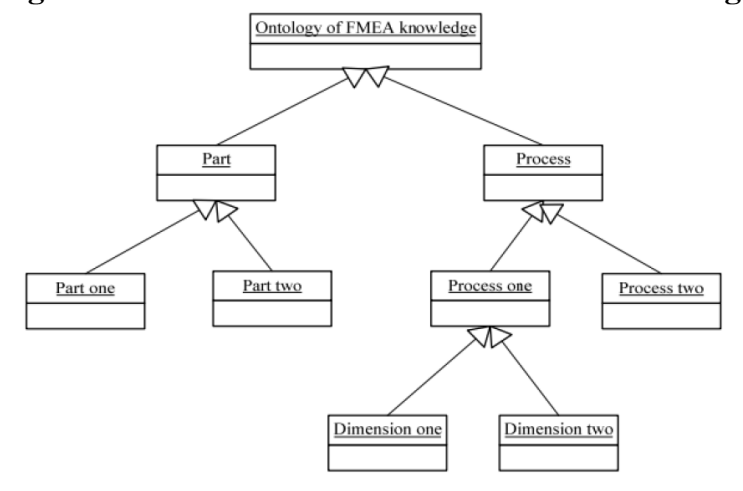

FMEA knowledge in the manufacturing system can be presented in a graphical notation following the components of manufacturing processes, process measurements, and all define a potential failure mode. This means that each component has a set of manufacturing processes, and each process has a set size requirements, thus, various failure modes may occur in this process. In the Figure 3 we describe this hierarchical relation graph of a specific process.

Based on analyses on FMEA knowledge the relation graph of failure modes contains a series of attributes and the specific attribute values, how is presented in Figure 4. 
Figure 4 Relation graph of failure modes

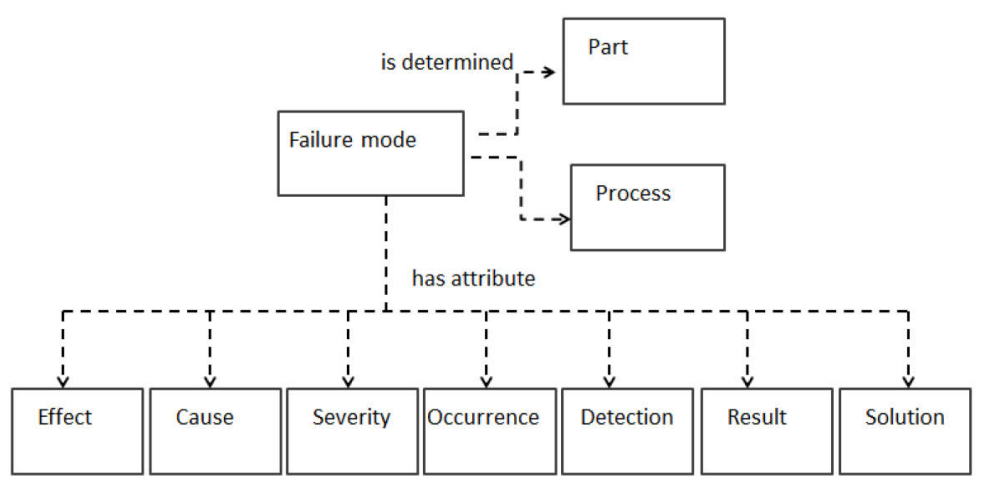

It was used Protégé, an open-source framework, to create and edit ontology and repository. With Protégé can be defined class and class hierarchy, attribute relations and attribute-value restriction and classattribute relations. The core modules of Protégé are mainly used to manage repository system, including knowledge representation and the establishment of knowledge hierarchy, acquisition and management.

Figure 5 FMEA ontology

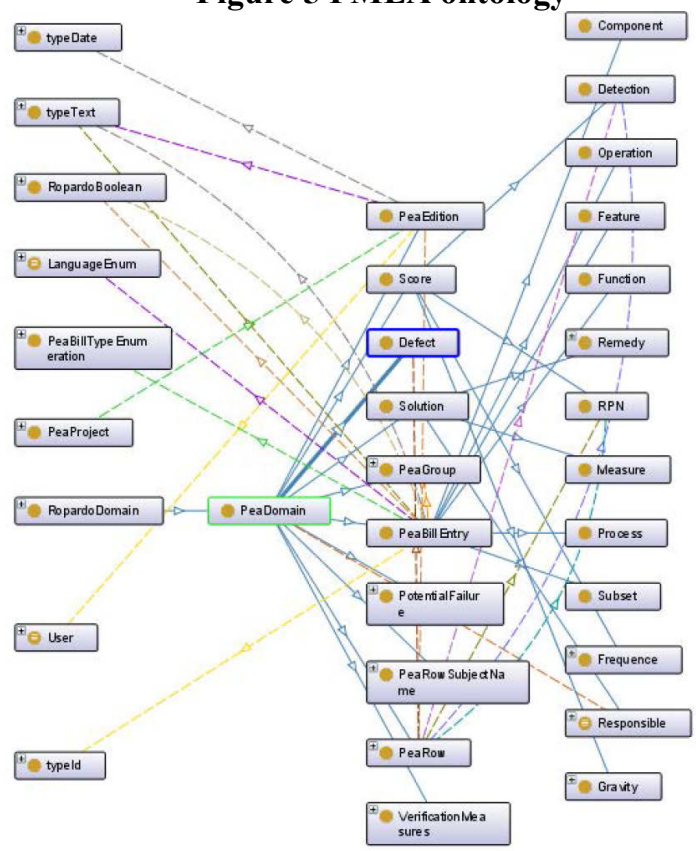

FMEA knowledge ontology was built by Protégé as following steps:

- Description of the concept hierarchy: using the upper and lower relationship in Protégé to show the relationship of class and subclass in FMEA knowledge, to hierarchize the knowledge of FMEA; For example, we have a "subclass_of" relationship between component and part;

- Adding property: the equivalent and correlation relationship from Protégé are used to show the internal structure of concepts; For example, each failure mode has: an effect, cause, severity number, occurrence, detection, result and solution property;

- Adding instance: the instance corresponding to one or more ontology concepts, have specific attributes and attribute values;

- Adding axiom: added some constraints among the concepts, instances and attributes of FMEA knowledge; such as function relation, inheritance relation, brotherhood relation, exclusive relation, partial order relation, etc. For example, constrain is each note must be from 1 to 10 .

And the result of this process can be shown in the Figure 5. 


\section{Conclusion}

The main objective of the research was to meet the practical need to acquire FMEA knowledge in manufacturing process, and this objective can be achieved by the system presented. This paper presents how the ontology can be used in manufacturing process for represent, acquire the FMEA related knowledge. In the next phase of the research it will be study how to identify the representative knowledge and retrieve this knowledge from repository.

\section{Acknowledgements}

Gabriela Simona Cândea was supported by project POSDRU/ CPP107/DMI1.5/S/76851, cofinanced by the European Social Found through Sectorial Operational Programme Human Resources Development 2007-2013.

\section{References}

- Cândea G.S. (2012), Knowledge, Knowledge Representation and Knowledge Management, $2^{\mathrm{ND}}$ Report of Doctoral Thesis

- Cândea G.S. (2012a), Intelligent system for assisting decision in planning and designing products and process, $3^{\mathrm{ND}}$ Report of Doctoral Thesis

- Dittmann, L., Rademacher, T., Zelewski, S. 2004. Performing FMEA using ontologies. In: Kleer, J.; Forbus, D. (eds.): 18th International Workshop on Qualitative Reasoning, Evanston, Illinois. pp. 209-216.

- Laaroussi,A., Fiès, B., Vankeisbelckt, R., Hans, J., Ontology-aided FMEA for construction products, in "Bringing ITC knowledge to work", $24^{\text {th }}$ W78 Conference Maribor 2007, 26.29.6.2007

- McDermott, R.E., Mikulak, R.J., Beauregard, M.R. (2008). The basics of FMEA. Productivity Press, USA

- Protégé, online: < http://protege.stanford.edu/> - ontology editor

- Stock, M., Stone, R. and Tumer, I. Y. (2003), Going Back in Time to Improve Design: The Function-Failure Design Method, Submitted to Proceedings of the 2003 ASME Design Engineering Technical Conference, Design Theory and Methodology Conference, Chicago, IL.

- Tague, N.R. (2004). The Quality Toolbox, Edition ASQ Quality Press, pp 236-240.

- UML, online: http://www.uml.org/

- Wirth, R., \& Berthold, B., \& Krämer, A., \& Peter, G. (1996). Knowledge-Based Support of System Analysis for Failure Mode and Effects Analysis. Engineering Applications of Artificial Intelligence, 9, 219-229 